\title{
AN ANALYSIS OF MANUFACTURING PRECISION OF INVOLUTE WORMS USING A KINEMATICAL MODEL
}

\author{
Csongor KELEMEN, ${ }^{1}$ Márton MÁTÉ ${ }^{2}$ \\ ${ }^{1}$ Sapientia Hungarian University of Transylvania, Faculty of Technical and Human Sciences, Târgu Mureș, \\ Romania, kelemen.e.csongor@stuent.ms.sapientia.ro \\ ${ }^{2}$ Sapientia Hungarian University of Transylvania, Faculty of Technical and Human Sciences, Târgu Mures, \\ Romania,mmate@ms.sapientia.ro
}

\begin{abstract}
The manufacturing precision of involute worms constitutes a major requirement. On the one hand, the worm constitutes the input element of the worm drive; secondly, the involute helical surface is the basic surface of an involute worm-hob. This paper presents an analytic comparison between the involute surfaces obtained using theoretical equations, kinematic simulation of the cutting and the surface charged with errors. The surface error is considered the distance along the normal direction to the theoretical surface, measured between this and the surface charged with simulated manufacturing errors. The main sources of errors are considered the center-error of the edge plane, the edge profile error and deviation of the axial feed direction from the axis of the worm. The theoretical results allow us to conclude that the influence of the edge profile error is the largest. It is followed by the parallelism error between the feed direction and the axis of the worm, and finally, the center error of the tool edge.
\end{abstract}

Keywords: involute worm, profile error, positioning error.

\section{The role of involute worm}

As is well known, manufacturing processes consist of a series of physical and chemical processes and, due to this, process parameters vary in a stochastic mode. As a consequence, the process parameter vertex slides away from the set point, even in the case of very high precision processes and modern infrastructure [1]. Manufacturing errors arise from the process. Constraining them between acceptable limits becomes possible only if the peculiarities of the operation are very well understood.

Here, it is necessary to emphasize the importance of manufacturing process modeling. Possible manufacturing errors must be anticipated, together with their qualitative and quantitative influences. Based on this, the success or the failure of a given operation can be predicted.

An eternal challenge in the field of machine part manufacturing is that of compliance regarding geometric precision. The harder the requirements referring to a given machine part, the more important the prediction of possible manufacturing errors; this constitutes an efficient procedure in order to discover the possible errors and develop the methods required to decrease or even eliminate them.

It is also well known that the manufacturing process of involute worms requires an infrastructure and corresponding control of exceptional precision. The load capacity, wear, and the functional heating result directly from the geometry of the bearing flanks and also from the relative position of these to the base surface [2, 3].

Involute worm flank surfaces are ruled surfaces, requiring relative low manufacturing costs and a not very sophisticated infrastructure. Although high power worm drives present profile modification [4, 5], involute worm drives inherit all the advantages of involute gear drives that justify their use in specific assemblies.

The study of the precision of the involute worm is also important because the flank surface is the 
origin surface of the involute gear hob edges. Intense research interest in this direction has been seen over the last two decades $[6,7,8]$.

The involute worm is commonly obtained by a threading operation, using a pair of straight edged threading cutters, where the flank meshing edges lie in two parallel planes that are tangential to the basic cylinder. This arrangement could potentially be a source of two types of error: the position of the edge plain relative to the basic cylinder, and the edge profile angle. The latter is the summative effect of the positioning and the manufacturing errors. A third source of error is supposed to arise from the parallelism deviation of the turning pad axis to the direction of the longitudinal motion.

Although the modern high-power worm drive's bearing surfaces are ground, here exists another possibility for fine cutting. This arises from the incredibly large variety of high performance cutting inserts, able to realize surface roughness and precision comparable with ground surfaces. Using them, the well-known expensive and environment-damaging grinding can be substituted with turning. Considering only the finishing operation, cutting forces will not increase over an acceptable limit, even in the case of hardened surface machining. Thus, the dimensions will be kept between the limits of tolerance. All these can be realized only if using modern machine-tools with increased rigidity.

The arguments given before, justify the necessity for the modeling of manufacturing errors.

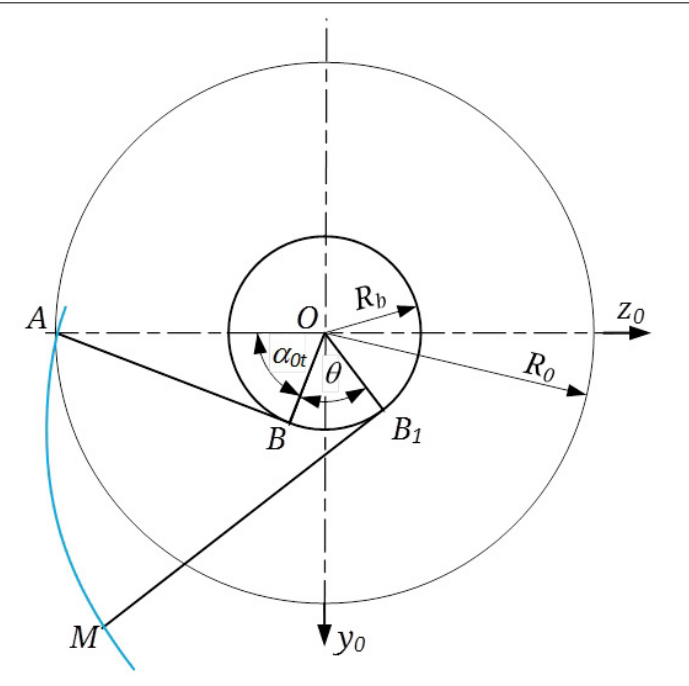

Figure 1. Definition of the involute curve in the case of an involute worm.

\section{The helical involute surface}

Equations of the helical involute surface will be given here using two different parametric forms: the first is based on mathematical meshing, while the second uses the geometric and the motion parameters of the real cutting edge.

\subsection{The theoretical involute helix surface}

The theoretical involute helix is meshed by an involute curve of plane $\left(y_{0} z_{0}\right)$ which executes a roto-translation about axis $x_{0}$ of a helix pitch that equals $m_{a x} \pi$. The parametric equations are written considering the geometric dependences shown in Figure 1. The form of equations differs from the classic one. Here the curve starts on the pitch circle, thereby omitting the unnecessary arc situated between the basic and the dedendum circle.

From Figure 1 the following parametric equations result:

$\int y_{0}(\theta)=R_{b}\left(\sin \left(\theta-i n v \alpha_{0 t}\right)-\theta \cos \left(\theta-i n v \alpha_{0 t}\right)\right)$

$\left\{z_{0}(\theta)= \pm R_{b}\left(\cos \left(\theta-i n v \alpha_{0 t}\right)+\theta \sin \left(\theta-i n v \alpha_{0 t}\right)\right)\right.$

The interval of the parameter $\theta$ is obtained by setting the limits of the radius length:

$R_{f} \leq R_{b} \sqrt{1+\theta^{2}} \leq R_{a} \Leftrightarrow \sqrt{\frac{R_{f}^{2}}{R_{b}^{2}}-1} \leq \theta \leq \sqrt{\frac{R_{a}^{2}}{R_{b}^{2}}-}$

Figure 2 shows the profile of the involute worm in a radial section.

Using the well-known dependences between the normal and axial module [4], the helix parameter can be primed as follows

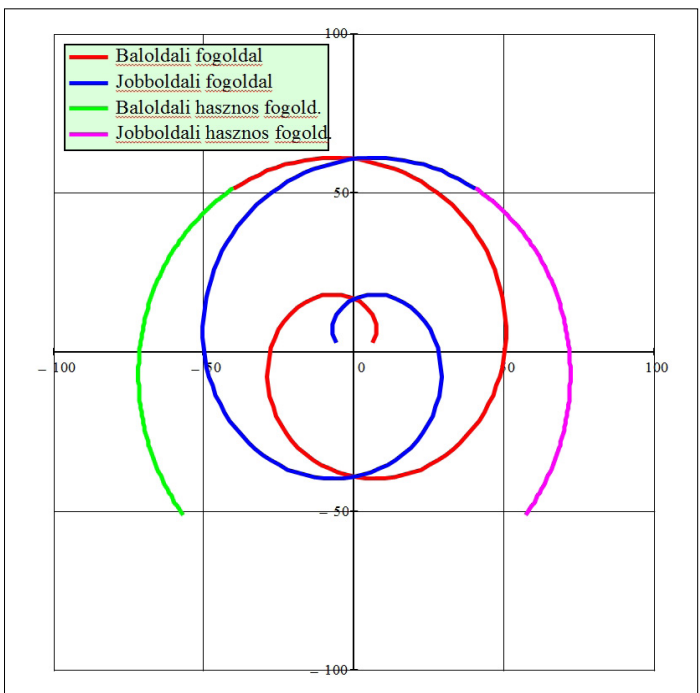

Figure 2. Radial section of an involute worm. 


$$
p=\frac{i}{2} \frac{m_{n}}{\cos \lambda_{0}}
$$

Omitting the computation, the surface obtained by applying a roto-translation of parameter $p$ to the involute (1), results in the following parametric equations:

$$
\left\{\begin{array}{c}
x_{0}(\theta, \varphi)=p \varphi \\
y_{0}(\theta, \varphi)=R_{b}(\sin E(\theta . \varphi)-\theta \cos E(\theta . \varphi)) \\
z_{0}(\theta, \varphi)= \pm R_{b}(\cos E(\theta . \varphi)+\theta \sin E(\theta . \varphi)) \\
E(\theta, \varphi)=\theta-i n v \alpha_{0 t}+\varphi
\end{array}\right.
$$

\subsection{The helical involute surface, meshed by the cutting edge}

The cutting-edge generated helical-involute parametric equations are obtained by applying to the cutting edges the same roto-translation of parameter $p$ given by (2), applied before. Cutting edges are situated in two parallel planes, each of them tangent to the basic cylinder. The mathematical form obtained here is of emphasized importance because it represents the errorless reference surface. It is compulsory to set the position of the edges in such way that the surfaces meshed by them include point $\mathrm{E}$ and its diametric opposite. Both points are situated on axis $z$ (Figure 3). These points also fit the pitch circle.

The profile angles are the angles comprised between the edges and a perpendicular to the worm axis $[1,7,8]$ :

$$
\alpha_{s} \equiv \lambda_{b}=\operatorname{arctg} \frac{p}{R_{b}}
$$

Because of the profile angle, a helical effect occurs (Figure 3), In order to overlap the mathematical and the kinematic involute surfaces, a translation of the edge along axis $x$ is necessary.

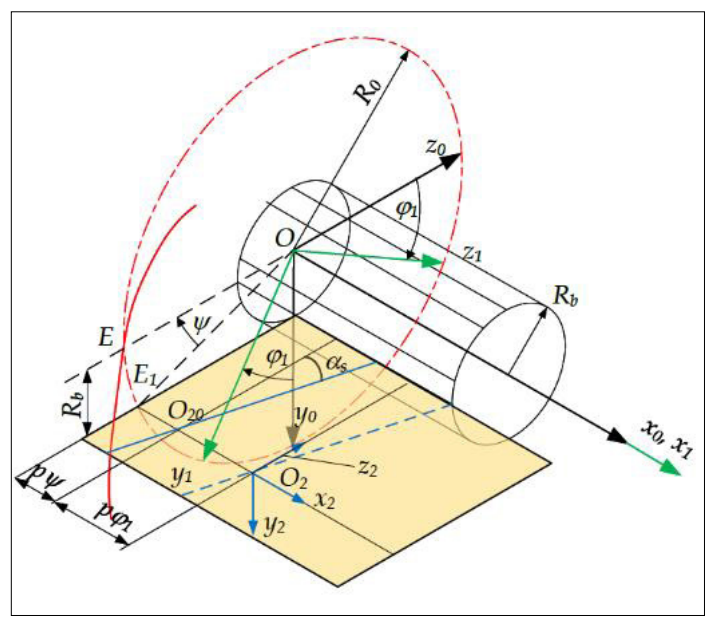

Figure 3. The positioning of the cutting edge.
The correction depends on angular correction $\psi$ expressed as:

$$
\psi=\arcsin \frac{R_{b}}{R_{0}}=\frac{\pi}{2}-\alpha_{0 t}
$$

The correction is based on a geometric peculiarity presented below. The left tooth gap surface (i.e. the right tooth surface) is meshed by the edge situated under the axis $x_{0}$, and it intersects the pitch cylinder in $O_{20}$.

The edge is at a distance $R_{b}$ from the $\left(x_{0} z_{0}\right)$ plane. Due to this, it must be rotated by the angle $\psi$ about the axis $O x_{0}$ to force point $O_{20}$ to overlap E.

The matrix form of the cutting edge related to the frame $\left(x_{2} y_{2} z_{2}\right)$ linked to the cutter body is as follows:

$$
\underline{\boldsymbol{r}}_{2}=\left(\begin{array}{lll}
u \frac{p}{R_{b}} & 0 & u
\end{array}\right)^{T}
$$

Moving the edge along the pitch helix of axis $x$ and parameter $p$ discussed before, it will mesh a helical surface described with the following parametric equations (here the calculus is omitted):

$$
\left\{\begin{array}{c}
x_{0}\left(u, \varphi_{1}\right)=p\left(\varphi_{1}+\frac{u}{R_{b}}\right) \\
y_{0}\left(u, \varphi_{1}\right)=R_{b}\left(\cos \varphi_{1}+\left(\operatorname{tg} \alpha_{0 t}-\frac{u}{R_{b}}\right) \sin \varphi_{1}\right) \\
z_{0}\left(u, \varphi_{1}\right)=R_{b}\left(\sin \varphi_{1}-\left(\operatorname{tg} \alpha_{0 t}-\frac{u}{R_{b}}\right) \cos \varphi_{1}\right)
\end{array}\right.
$$

\subsection{The comparison of the theoretical heli- cal involute surfaces}

The theoretically perfect, errorless helical surfaces are coincident. This can be mathematically proven by deducting a bijective dependence $(\varphi, \theta) \leftrightarrow\left(\varphi_{1}, u\right)$ between the independent parameters of coordinate functions (4) and (8).

Equalizing the x-coordinates, results in

$$
\varphi=\varphi_{1}+\frac{u}{R_{b}}
$$

By the other side, if equalizing the polar radii we get:

$$
\theta-i n v \alpha_{0 t}+\varphi= \pm\left(\operatorname{tg} \alpha_{0 t}-\frac{u}{R_{b}}\right)
$$

Considering the right side with the minus sign and substituting expression (9) in the left side, equation (10) takes the following form:

$$
\theta+\alpha_{0 t}+\varphi_{1}=0 \Leftrightarrow \theta=-\left(\alpha_{0 t}+\varphi_{1}\right)
$$

The last equation can be considered an interesting approach of the equivalence of the considered helical surface meshing procedures. The role of the parameter $\theta$ in expression (4) consists 
in the generating of the involute curve as can be observed from equations (1). On the other hand, parameter $\varphi_{1}$ from equation (8) expresses the quantum of the rotational component of the helical generating motion. Despite the fact that the functional role of the mentioned parameters differs, the correspondence can be accepted. In the first case where the involute is used as generating curve, all surface points corresponding to an arbitrary value of the rotation parameter are comprised in the same radial plane. In the second case, where the generating curve is the cutting edge, surface points having the same rotational parameter value are situated on the edge, not in a common radial plane as before.

On the other hand, condition (9) expresses the equality of the x-coordinates. Thus, the considered points are constrained to fit the same radial plane. Furthermore, these points can overlap if, and only if, expression (11) involving $\theta$ and $\varphi_{1}$ is true.

Conditions (9) and (10) involving surface parametric equations (4) and (8) were numerically computed. The simulation shows a difference of a $10^{-15}$ magnitude between the $x, y, z$ coordinates computed with formulae (4) and (8).

\section{The simulation of the manufacturing errors}

The manufacturing errors arise from the positioning of the cutter, the geometric errors of the machine tool and the edge profile error. The effect of each of them is separately studied because they are independent statistical variables.

\subsection{The center error of the cutter}

The center error of the cutter can be defined as the deviation of the distance between the rotation axis and the edge holding plane, from the basic radius value. To express this deviation a weighting coefficient $f$ is introduced. Applying it to the edge coordinate $y$ expressed by matrix (7), value $\mathrm{R} \_\mathrm{b}$ is replaced with $f R_{b}$. If $f>0$, the edge is pushed down, while for $f<0$, it is raised up.

The parametric coordinates result in the following matrix equation:

$$
\begin{aligned}
\underline{\boldsymbol{r}}_{0}=\boldsymbol{M}_{02} \underline{\boldsymbol{r}}_{2} & \boldsymbol{M}_{02}=\left(\begin{array}{cccc}
1 & 0 & 0 & p \varphi \\
0 & \cos \varphi & -\sin \varphi & a_{24} \\
0 & \sin \varphi & \cos \varphi & a_{34} \\
0 & 0 & 0 & 1
\end{array}\right)
\end{aligned}
$$

$$
\begin{aligned}
& a_{24}=R_{b} \cos \varphi+\sqrt{R_{0}^{2}-R_{b}^{2} \sin \varphi} \\
& a_{34}=R_{b} \sin \varphi-\sqrt{R_{0}^{2}-R_{b}^{2}} \cos \varphi \\
& \underline{\boldsymbol{r}}_{2}=\left(\begin{array}{llll}
u \frac{p}{R_{b}} & f R_{b} & u & 1
\end{array}\right)^{T}
\end{aligned}
$$

The helical surface written with the expressions (12)..(14), becomes a perfect helical involute surface only if parameter $f=0$. The border positions of the real helical surface, for the maximum value of the setting error can be obtained from $f R_{b}=\Delta h$ equivalent with the $f$-interval $-\frac{\Delta h}{R_{b}} \leq f \leq \frac{\Delta h}{R_{b}}$.

\subsection{The parallelism error}

The parallelism error is defined as the deviation between the trajectory of the longitudinal feed motion and the rotation axis of the turning machine, for a motion length of $L=1000 \mathrm{~mm}$. In this paper the deviation is particularized by considering the trajectory of the feed line included in the horizontal plane $(x z)$. Thus, the trajectory line includes, with the axis, an angle $\beta$ of magnitude $\operatorname{tg} \beta \approx \beta=\delta_{p} / 10^{3}$. A $\delta_{p}=100 \mu \mathrm{m}$ parallelism deviation value occurs when the machine tool reaches its final wear state. This state leads to $\beta \approx 1 \cdot 10^{-4} \mathrm{rad}$, namely 20.62 sexadecimal seconds.

Admitting the described feed trajectory deviation, the last column of matrix $\mathbf{M}_{02}$ becomes:

$$
\left(\begin{array}{c}
p \varphi \\
-R_{b} \cos \varphi+\left(\sqrt{R_{0}^{2}-R_{b}^{2}}+p \varphi \beta\right) \sin \varphi \\
-R_{b} \sin \varphi-\left(\sqrt{R_{0}^{2}-R_{b}^{2}}+p \varphi \beta\right) \cos \varphi \\
1
\end{array}\right)
$$

The $\mathrm{R}^{3}$ subspace of the helical involute manifold results for $-10^{-4} \leq \beta \leq 10^{-4}$ In this model the origin of the worm's frame is placed in the middle point of its length. If the tool setting is computed considering the limiting frontal plane of the worm, the frame's origin must be translated here.

\subsection{The profile error}

Profile error is defined as the maximum value of the distance between the real edge point to the theoretical straight edge line (Figure 4). Profile error can be perceived either as the deviation of a concave or convex line from its tangent in the reference point, where the tangent is the theoretical edge direction, or as a straight line whose profile angle differs from the theoretical, but it intersects the theoretical edge segment in the reference 
point. The reference point discussed before is almost the edgepoint acting on the pitch cylinder. The profile error is assimilated with the quantity $F_{\alpha}$ defined in gear precision standard and here it is considered for an N8 precision class [9].

In the first case the error laden edge is expressed using a polynomial of $3^{\text {rd }}$ degree. The requirements imposed on this refer to the deviation that must be smaller than the chosen $F_{\alpha}$ value, considering the useful length of edge $2.25 m_{n} / \cos \alpha_{s}$. Another restriction imposed on the polynomial consists in its tangency to the theoretical edge line, in the origin (Figure 4).

The geometric constraints presented before result in at the most six algebraic equations, thus, the simplest polynomial here is a polynomial of $3^{\text {rd }}$ degree whose graphic crosses the origin; let's define it parametrically, using the following functions:

$$
\left\{\begin{array}{l}
x_{2}(u)=a_{1} u^{3}+b_{1} u^{2}+c_{1} u \\
z_{2}(u)=a_{2} u^{3}+b_{2} u^{2}+c_{2} u
\end{array}\right.
$$

(the free term of the polynomial is missing because in the origin $u=0$.)

The limits of $u$ are (Figure 3 ):

$$
\begin{aligned}
& u_{1}=-\left(\sqrt{R_{a}^{2}-R_{b}^{2}}-\sqrt{R_{0}^{2}-R_{b}^{2}}\right) ; \\
& u_{2}=\sqrt{R_{0}^{2}-R_{b}^{2}}-\sqrt{R_{f}^{2}-R_{b}^{2}} \\
& u_{1} \leq u \leq u_{2}
\end{aligned}
$$

The profile error is measured perpendicular to the theoretical edge line. Accepting that the inflexion point is missing (i.e. the real edge line is either concave or convex), the coordinates of the endpoints for the convex edge can be written as:

$$
\begin{aligned}
& \left\{\begin{array}{l}
x_{21}=x_{2}\left(u_{1}\right)=u_{1} \operatorname{tg} \alpha_{s}-F_{\alpha} \cos \alpha_{s} \\
z_{21}=z_{2}\left(u_{1}\right)=u_{1}+F_{\alpha} \sin \alpha_{s}
\end{array}\right. \\
& \left\{\begin{array}{l}
x_{22}=x_{2}\left(u_{2}\right)=u_{2}-F_{\alpha} \cos \alpha_{s} \\
z_{21}=z_{2}\left(u_{1}\right)=u_{2}+F_{\alpha} \sin \alpha_{s}
\end{array}\right.
\end{aligned}
$$

In the same way the concave edge endpoint coordinates result in:

$$
\begin{aligned}
& \left\{\begin{array}{l}
x_{21}=x_{2}\left(u_{1}\right)=u_{1} \operatorname{tg} \alpha_{s}+F_{\alpha} \cos \alpha_{s} \\
z_{21}=z_{2}\left(u_{1}\right)=u_{1}-F_{\alpha} \sin \alpha_{s}
\end{array}\right. \\
& \left\{\begin{array}{l}
x_{22}=x_{2}\left(u_{2}\right)=u_{2}+F_{\alpha} \cos \alpha_{s} \\
z_{21}=z_{2}\left(u_{1}\right)=u_{2}-F_{\alpha} \sin \alpha_{s}
\end{array}\right.
\end{aligned}
$$

The condition of tangency in the origin becomes:

$$
\operatorname{tg} \alpha_{s}=\left.\frac{\dot{z}}{\dot{x}}\right|_{u=0}=\frac{c_{2}}{c_{1}}
$$

From condition (20) results obviously in:

$$
c_{1}=\cos \alpha_{s} ; c_{2}=\sin \alpha_{s} \text {, }
$$

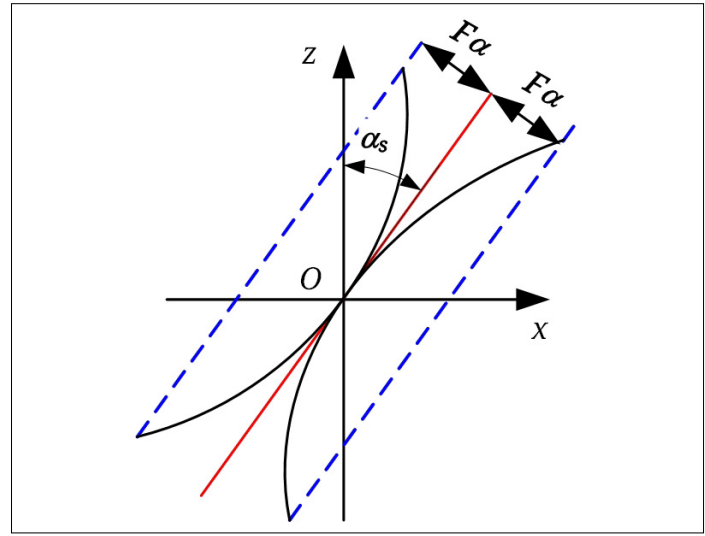

Figure 4. The definition of the profile error

Considering this together with expressions (16), (18) and (19), a linear system with unknowns $a_{1}, a_{2}, b_{1}, b_{2}$ builds up. Its solution are the coefficients of the $3^{\text {rd }}$ degree polynomial.

The real helical surface equation results by using in matrix equation (12) the homogenous coordinate vector $\underline{\boldsymbol{r}_{2}}=\left(\begin{array}{llll}x_{2}(u) & 0 & z_{2}(u) & 1\end{array}\right)^{T}$.

\section{Numerical evaluation}

Numerical evaluation of the model presented before follows the steps emphasized below:

- writing the equations of the real helical surface laden with the considered type of error, followed by the computing of surface point coordinates inside a fixed domain $\left(u_{1}, u_{2}\right) \times\left(\varphi_{1}, \varphi_{2}\right)$;

- computing the $x_{0}^{*}$ coordinate of the pitch helix point comprised in the plane $\left(x_{0} z_{0}\right)$;

-performing the translation of the coordinates along the helix axis, given by $x^{\prime}=x_{0}-x_{0}^{*}$;

- computing the length of the normal segment between the theoretical and the real surface- this is the error.

The proofing was performed for an involute worm of $m_{n}=5 \mathrm{~mm}$ normal module, $\mathrm{i}=1$ number of teeth, $\lambda_{0}=4^{\circ}$ pith helix angle.

First the center error was analyzed, considering the errors defined by $f=0.1$ and $f=0.2$. The distribution of the errors is shown in Figure 5 .

On the basis of figure 5 it can be stated that by doubling the center error value the normal error increases more than two times, thus the dependence is not linear. Although it must be emphasized that the maximal normal error value, for a center error of $f=0.2$-re, meaning $\Delta h=0.2 R_{b}=$ $2.698 \mathrm{~mm}$ reaches only $\Delta_{n h}=1.631 \mu \mathrm{m}$. Inspecting the shape of the error distribution it can be stated that the error is positive signed on the addendum, 


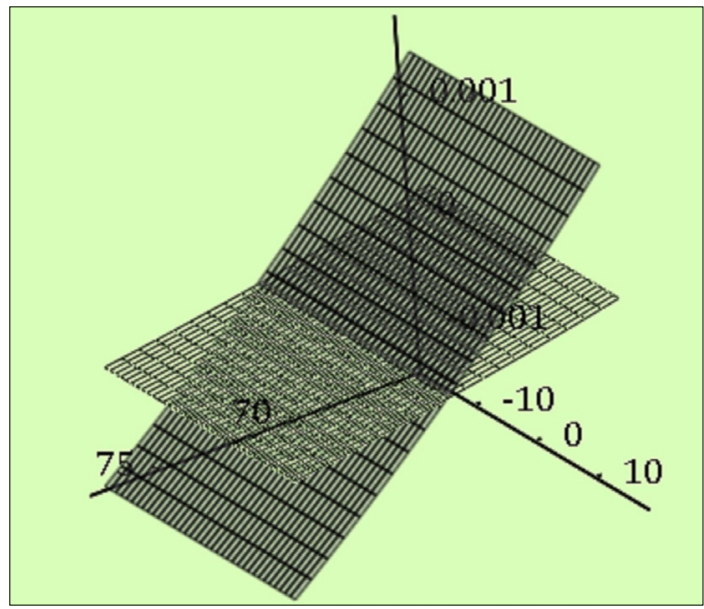

Figure 5. The distribution of the center error.

and negative on the dedendum cylinder. Positive error means that the real helical surface is larger than the theoretical, including it, while negative error means that an undercut occurs.

Practical experience shows that the maximum center error value in the case of worm threading does not exceed $0.1 \mathrm{~mm}$, thus normal error is limited to a submicronic magnitude.

Starting from a N8 precision class and the corresponding profile error value of $F_{\alpha}=27 \mu \mathrm{m}$, edge profile error induced surface normal errors were computed for the same worm. The repartition of the computed value is shown in Figure 6.

The effect of the concave deviation of the cutting edge results in the surface located below, while the convex deviation in the surface located above. It must be emphasized that the error value on the pitch cylinder is zero. This proves the correctness of the computation and the model, while tangency of the theoretical and the error laden edge was imposed.

In contrast to the center error causing normal error repartition, here the error keeps its sign. The absolute values shown an approximately parabolic evolution.

The cutting edge's concave deviance produces a real helical surface that includes the theoretical involute helical worm surface. Conversely, a convex edge deviance results in a real helical surface that undercuts the theoretical. The common entity of all three is the pitch helix.

In this case, the maximum value of the normal error does not exceed $\Delta_{n p} \approx 13 \mu \mathrm{m}$, which is only half of the admissible edge profile deviation.

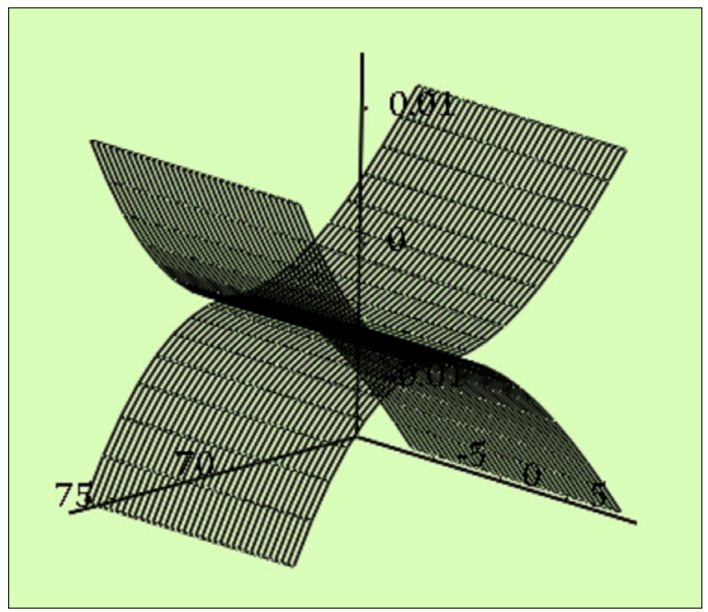

Figure 6. The normal error repartition caused by the edge profile error.

Results obtained by applying the parabolic model are useful even in the case of a linear edge with angular deviation. In this case the theoretical edge is placed in the middle of the tolerance field, and thus, the maximum error occurs when the real edge lies on the diagonal.

It can be concluded that the previous model must set up for $F_{a} / 2$ value. The expected normal profile error magnitude becomes $\Delta_{n p} \approx 0.25 F_{\alpha}=7 \mu \mathrm{m}$.

The cumulative error value is computed considering the center and the profile errors as independent statistical variables. The sign of the error is here omitted; only absolute values are taken into consideration.

Let us denote with $\xi$ the statistical variable defining the normal error caused by the center error, and with $\eta$ the error caused by the edge profile. The variance extends within the zero and the maximum error value, thus

$$
\left\{\begin{array}{l}
0 \leq \xi \leq \Delta_{n h} \\
0 \leq \eta \leq \Delta_{n p}
\end{array}\right.
$$

Admitting a reasonable approach, it can be supposed that the average value of the cumulative error is:

$$
\bar{\zeta}=\bar{\xi}+\bar{\eta}=\frac{13}{2}+\frac{1,631}{2} \approx 7,35 \mu m
$$

In a same way a good approach of the standard deviation can be considered the $1 / 6$ part of the variance [10]. Thus, the standard deviation of the cumulative error becomes:

$$
\begin{aligned}
& \sigma_{\zeta}=\sqrt{\sigma_{\xi}^{2}+\sigma_{\eta}^{2}}=\frac{1}{6} \sqrt{\Delta_{n h}^{2}+\Delta_{n p}^{2}}= \\
& =\frac{1}{6} \sqrt{13^{2}+1,631^{2}} \approx 2,2 \mu \mathrm{m}
\end{aligned}
$$


In the spite of the fact that the composition of two normally distributed statistical variables results in an exponential distribution [10], let us consider $\zeta$ normal distributed. The differences can be neglected as well, as it was proven by many industrial applications. The statistical computing model of the machining allowances [11] is built up admitting the same hypotheses.

Admitting these the maximum value of the $\mathrm{cu}$ mulative normal error becomes:

$$
\Delta_{\Sigma}=\bar{\zeta}+3 \sigma_{\zeta}=7.35+3 \cdot 2.2=13.95 \mu \mathrm{m}
$$

\section{Conclusions}

Mathematical models presented in this paper can be used for the estimation of the expected manufacturing error value, in the case of involute worm threading,

The presented method is notably important while having knowledge upon the geometric precision of the manufacturing infrastructure. With this model it can be decided if a given involute worm can or cannot be realized within the prescribed limits of tolerance.

The worm error sources were supposed to have originated from the center positioning error of the edge, the profile error of the cutting edge and the parallelism error of the machine tool. The last was neglected, thus, the cumulative error was computed as the sum of the center and the profile errors.

The center error and the profile error were considered as independent statistic variables.

The error was defined as the normal distance between the real and the errorless involute helix surfaces.

In our perception, error is plus signed if the real surface covers the theoretical, and negative if undercuts it.

The computing of the normal error was realized within a synthetic geometric model that was not presented here.

The numerical evaluation has proven that the center error has the least impact on the cumulative error, while its rate is only $11,69 \%$ of the cumulative error- even in the case of considering exaggerated values of the tool center error.

In conclusion it can be stated with a good approximation that the involute worm manufacturing cumulative error equals half of the profile error.
The error of the threading kinematic chain was neglected because the axial extent of the worm will never exceed 6-8 integral pitches. On that length, the pitch errors of the leading screw, as well as the parallelism error influences are not relevant. It must be mentioned here that modern, numerical driven machine's kinematic chains present pitch error of micronic magnitude. .

\section{References}

[1] Dudas I., Varga G., Banyai K.: Holonic manufacturing system for production of different sophisticated surfaces. Proceedings of the IASTED International Conference on Modelling, Simulation and Optimization (2004) 72-75.

[2] Balajti Zs.: Examination and adjustment of the bearing pattern in case of helicoid drive. 8th CIRP Conference on High Performance Cutting, Budapest, Hungary, June 25-27. 2018. Procedia CIRP, 77 (2018) 267-270.

[3] Balajti Zs., Dudás I.: The Monge Theorem and Its Application in Engineering Practice. The International Journal of Advanced Manufacturing Technology. Article 9763, Springer, London (2016). https://doi.org/10.1007/s00170-016-9763-1

[4] Dudas I.: The theory and practice of worm gear drives. Penton Press, London, 2000.

[5] I Tsay C. B., Tseng J. T.: Undercutting and contact characteristics of cylindrical gears with curvilinear shaped teeth generated by hobbing. Journal of Mechanical Design, 128/3. (2006) 634-643.

[6] Mohan L. V.: Geometrical aspects of double enveloping worm gear drive. Mechanism and Machine Theory, 44, (2009) 2053-2065.

[7] Radzevich S. P.: A way to improve the accuracy of hobbed involute gears. Journal of Mechanical design, 129/10. (2007), 1076-1085.

https://doi.org/10.1115/1.2761919

[8] Radzevich S. P.: Investigation of the tooth geometry of a hob for manufacturing of involute gears (in Tool-in-Use References System). Journal of Manufacturing Science and Engineering, 129/4. (2007), 750-759.

[9] KG Stock Gears: Gear Technical Data. 5.7 Precision of Spur and Helical gears. Letöltés: 2021. 04. 06.

https://www.kggear.co.jp/en/wp-content/themes/ bizvektor-global-edition/pdf/5.7_Precision-ofSpur-and-Helical-gears_TechnicalData_KGSTOCKGEARS.pdf.

[10] Cseke V.: A valószínűségszámítás alapjai. Dacia Könyvkiadó, Kolozsvár, 1982.

[11] Drăghici G.: Tehnologia Construcțiilor de mașini. E.D.P., Bukarest, 1985. 